\title{
Existence of Positive Solutions for Third-Order $m$-Point Boundary Value Problems with Sign-Changing Nonlinearity on Time Scales
}

\author{
Fatma Tokmak and Ilkay Yaslan Karaca \\ Department of Mathematics, Ege University, Izmir, 35100 Bornova, Turkey \\ Correspondence should be addressed to Ilkay Yaslan Karaca, ilkay.karaca@ege.edu.tr
}

Received 2 August 2012; Accepted 8 November 2012

Academic Editor: Yongfu Su

Copyright (c) 2012 F. Tokmak and I. Y. Karaca. This is an open access article distributed under the Creative Commons Attribution License, which permits unrestricted use, distribution, and reproduction in any medium, provided the original work is properly cited.

\begin{abstract}
A four-functional fixed point theorem and a generalization of Leggett-Williams fixed point theorem are used, respectively, to investigate the existence of at least one positive solution and at least three positive solutions for third-order $m$-point boundary value problem on time scales with an increasing homeomorphism and homomorphism, which generalizes the usual $p$-Laplacian operator. In particular, the nonlinear term $f(t, u)$ is allowed to change sign. As an application, we also give some examples to demonstrate our results.
\end{abstract}

\section{Introduction}

The theory of time scales was introduced by Hilger in his Ph.D. thesis in 1990 [1]. Theoretically, this new theory not only unifies continuous and discrete equations, but has also exhibited much more complicated dynamics on time scales. Moreover, the study of dynamic equations on time scales has led to several important applications, for example, insect population models, biology, neural networks, heat transfer, and epidemic models (see $[2,3]$ and references therein). Some preliminary definitions and theorems on time scales also can be found in books [2,3], which are excellent references for calculus on time scales.

Multipoint boundary value problem (BVP) arises in a variety of different areas of applied mathematics and physics [4]. The study of multipoint BVPs for linear second-order ordinary differential equations was initiated by Ilin and Moiseev [5], since then many authors studied more general nonlinear multipoint boundary value problems $([6,7]$ and references therein). Recently, when $\varphi_{p}$ is $p$-Laplacian operator, that is, $\varphi_{p}(u)=|u|^{p-2} u(p>1)$ and the nonlinear term does not depend on the first-order derivative, the existence problems of positive solutions of boundary value problems have attracted much attention. One can notice 
that the oddness of the $p$-Laplacian operator is key to the proof. However, in the operator $\phi$ is not necessary odd, so it improves and generalizes the $p$-Laplacian operator.

There are few works that investigate the existence of positive solutions by using four functionals fixed point theorem [8] or a generalization of Leggett-Williams fixed point theorem [9]. Avery et al. [8] present a four-functional fixed point theorem, which is a major generalization of the original Leggett-Williams fixed point theorem [10]. To our knowledge, this fixed point theorem so far has only been used by Sun et al. [11]. By using the fourfunctional fixed point theorem and five-functional fixed point theorem, they obtain the existence criteria of at least one positive solution and three positive solutions for the nonlocal BVP with $p$-Laplacian. Liu and Sun [12] discussed the existence of at least three and arbitrary odd number positive solutions for $m$-point boundary value problems on time scale. They used a generalization of Leggett-Williams fixed point theorem [9].

There have been extensive studies on BVP with sign-changing nonlinearity [13-17]. Most of these results are obtained by using the fixed point theorem on cone [18, 19]. To the best of our knowledge, positive solutions of third-order $m$-point BVP for an increasing homeomorphism and homomorphism with sign-changing nonlinearity on time scales by using four functionals fixed point theorem [8] or a generalization of Leggett-Williams fixed point theorem [9] have not been considered till now. Our aim in this paper is to fill the gap.

In this paper, motivated by above results, we consider the existence of at least one or three positive solutions of the following third-order $m$-point boundary value problem (BVP) on time scales

$$
\begin{gathered}
\left(\phi\left(u^{\Delta \nabla}(t)\right)\right)^{\nabla}+a(t) f(t, u(t))=0, \quad t \in[0, T]_{\mathbb{T}} \\
u(0)=\sum_{i=1}^{m-2} b_{i} u\left(\xi_{i}\right), \quad u^{\Delta}(T)=0, \quad \phi\left(u^{\Delta \nabla}(0)\right)=\sum_{i=1}^{m-2} c_{i} \phi\left(u^{\Delta \nabla}\left(\xi_{i}\right)\right),
\end{gathered}
$$

where $\phi: \mathbb{R} \rightarrow \mathbb{R}$ is an increasing homeomorphism and homomorphism with $\phi(0)=0$. A projection $\phi: \mathbb{R} \rightarrow \mathbb{R}$ is called an increasing homeomorphism and homomorphism if the following conditions are satisfied.

(i) If $x \leq y$, then $\phi(x) \leq \phi(y)$, for all $x, y \in \mathbb{R}$;

(ii) $\phi$ is continuous bijection and its inverse mapping is also continuous;

(iii) $\phi(x y)=\phi(x) \phi(y)$, for all $x, y \in \mathbb{R}$.

We will assume that the following conditions are satisfied throughout this paper:

(H1) $b_{i}, c_{i} \in[0,+\infty), 0<\xi_{1}<\xi_{2}<\cdots<\xi_{m-2}<\rho(T), 0<\sum_{i=1}^{m-2} b_{i}<1,0<\sum_{i=1}^{m-2} c_{i}<1$;

(H2) $f:[0, T]_{\mathbb{T}} \times[0,+\infty) \rightarrow(-\infty,+\infty)$ is continuous, $a \in \mathcal{C}_{\mathrm{ld}}\left([0, T]_{\mathbb{T}},[0,+\infty)\right)$, and there exist $t_{0} \in[0, T]_{\mathbb{T}}$ such that $a\left(t_{0}\right)>0$;

(H3) $\zeta=\min \{t \in \mathbb{T}: t>T / 2\}$ exist.

By using a four-functional fixed point theorem [8], we establish the existence of at least one positive solution, and by using a generalization of Leggett-Williams fixed point theorem [9], we establish the existence of at least three positive solutions for the above boundary value problem. In particular, the nonlinear term $f(t, u(t))$ is allowed to change sign. The remainder of this paper is organized as follows. Section 2 is devoted to some preliminary lemmas. We give and prove our main results in Section 3. Finally, in Section 4, we give two examples to demonstrate our results. 


\section{Preliminary Lemmas}

In this section, we present some definitions and lemmas, which will be needed in the proof of the main results.

Lemma 2.1 (see [13]). If condition (H1) holds, then for $h \in \mathcal{C}_{\mathrm{ld}}[0, T]_{\mathbb{T}}$, the boundary value problem $(B V P)$,

$$
\begin{gathered}
u^{\Delta \nabla}+h(t)=0, \quad t \in[0, T]_{\mathbb{T}}, \\
u(0)=\sum_{i=1}^{m-2} b_{i} u\left(\xi_{i}\right), \quad u^{\Delta}(T)=0,
\end{gathered}
$$

has a unique solution

$$
u(t)=\int_{0}^{t}(T-s) h(s) \nabla s+\frac{\sum_{i=1}^{m-2} b_{i} \int_{0}^{\xi_{i}}(T-s) h(s) \nabla s}{1-\sum_{i=1}^{m-2} b_{i}} .
$$

Lemma 2.2. If condition (H1) holds, then for $h \in \mathcal{C}_{\mathrm{ld}}[0, T]_{\mathbb{T}}$, the boundary value problem (BVP),

$$
\begin{gathered}
\left(\phi\left(u^{\Delta \nabla}(t)\right)\right)^{\nabla}+h(t)=0, \quad t \in[0, T]_{\mathbb{T}} \\
u(0)=\sum_{i=1}^{m-2} b_{i} u\left(\xi_{i}\right), \quad u^{\Delta}(T)=0, \quad \phi\left(u^{\Delta \nabla}(0)\right)=\sum_{i=1}^{m-2} c_{i} \phi\left(u^{\Delta \nabla}\left(\xi_{i}\right)\right),
\end{gathered}
$$

has a unique solution

$$
u(t)=\int_{0}^{t}(s-T) \phi^{-1}\left(-\int_{0}^{s} h(r) \nabla r+D\right) \nabla s+\frac{\sum_{i=1}^{m-2} b_{i} \int_{0}^{\xi_{i}}(s-T) \phi^{-1}\left(-\int_{0}^{s} h(r) \nabla r+D\right) \nabla s}{1-\sum_{i=1}^{m-2} b_{i}},
$$

where $D=-\left(\sum_{i=1}^{m-2} c_{i} \int_{0}^{\xi_{i}} h(r) \nabla r\right) /\left(1-\sum_{i=1}^{m-2} c_{i}\right)$.

Proof. Integrating both sides of equation in $(2.3)$ on $[0, t]$, we have

$$
\phi\left(u^{\Delta \nabla}(t)\right)=\phi\left(u^{\Delta \nabla}(0)\right)-\int_{0}^{t} h(r) \nabla r
$$

So,

$$
\phi\left(u^{\Delta \nabla}\left(\xi_{i}\right)\right)=\phi\left(u^{\Delta \nabla}(0)\right)-\int_{0}^{\xi_{i}} h(r) \nabla r .
$$


By boundary value condition $\phi\left(u^{\Delta \nabla}(0)\right)=\sum_{i=1}^{m-2} c_{i} \phi\left(u^{\Delta \nabla}\left(\xi_{i}\right)\right)$, we have

$$
\phi\left(u^{\Delta \nabla}(0)\right)=-\frac{\sum_{i=1}^{m-2} c_{i} \int_{0}^{\xi_{i}} h(r) \nabla r}{1-\sum_{i=1}^{m-2} c_{i}}
$$

By (2.5) and (2.7), we know

$$
u^{\Delta \nabla}(t)=\phi^{-1}\left(-\frac{\sum_{i=1}^{m-2} c_{i} \int_{0}^{\xi_{i}} h(r) \nabla r}{1-\sum_{i=1}^{m-2} c_{i}}-\int_{0}^{t} h(r) \nabla r\right)
$$

This together with Lemma 2.1 implies that

$$
u(t)=\int_{0}^{t}(s-T) \phi^{-1}\left(-\int_{0}^{s} h(r) \nabla r+D\right) \nabla s+\frac{\sum_{i=1}^{m-2} b_{i} \int_{0}^{\xi_{i}}(s-T) \phi^{-1}\left(-\int_{0}^{s} h(r) \nabla r+D\right) \nabla s}{1-\sum_{i=1}^{m-2} b_{i}},
$$

where $D=-\left(\sum_{i=1}^{m-2} c_{i} \int_{0}^{\xi_{i}} h(r) \nabla r\right) /\left(1-\sum_{i=1}^{m-2} c_{i}\right)$. The proof is complete.

Lemma 2.3. Let condition $(H 1)$ hold. If $h \in \mathcal{C}_{\mathrm{ld}}[0, T]_{\mathbb{T}}$ and $h(t) \geq 0$, then the unique solution $u(t)$ of (2.3) satisfies

(i) $u(t) \geq 0, t \in[0, T]_{\mathbb{T}}$,

(ii) $\inf _{t \in[0, T]_{\mathbb{T}}} u(t) \geq \sigma\|u\|$,

where $\sigma=\sum_{i=1}^{m-2} b_{i} \xi_{i} /\left(T-\sum_{i=1}^{m-2} b_{i}\left(T-\xi_{i}\right)\right),\|u\|=\sup _{t \in[0, T]_{\mathbb{T}}}|u(t)|$.

Proof. (i) Let $\psi_{0}(s):=\phi^{-1}\left(-\int_{0}^{s} h(r) \nabla r+D\right)$. Consider the following equation:

$$
-\int_{0}^{s} h(r) \nabla r+D=-\int_{0}^{s} h(r) \nabla r-\frac{\sum_{i=1}^{m-2} c_{i} \int_{0}^{\xi_{i}} h(r) \nabla r}{1-\sum_{i=1}^{m-2} c_{i}} \leq 0 .
$$

So, we have $\psi_{0}(s) \leq 0$. Hence $u(t) \geq 0, t \in[0, T]_{\mathbb{T}}$.

(ii) By $u^{\Delta \nabla}(t)=\phi^{-1}\left(-\int_{0}^{t} h(r) \nabla r+D\right) \leq 0$, we can know that the graph of $u(t)$ is concave down on $(0, T)_{\mathbb{T}}$ and $u^{\Delta}(t)$ is nonincreasing on $[0, T]_{\mathbb{T}}$. This together with the assumption that the boundary condition $u^{\Delta}(T)=0$ implies that $u^{\Delta}(t) \geq 0$ for $t \in[0, T]_{\mathbb{T}}$. This implies that

$$
\|u\|=u(T), \quad \min _{t \in[0, T]_{T}} u(t)=u(0) .
$$

For all $i \in\{1,2, \ldots, m-2\}$, we have from the concavity of $u$ that

$$
\frac{u\left(\xi_{i}\right)-u(0)}{\xi_{i}} \geq \frac{u(T)-u(0)}{T},
$$


that is,

$$
u\left(\xi_{i}\right)-u(0)+\frac{\xi_{i}}{T} u(0) \geq \frac{\xi_{i}}{T} u(T)
$$

This together with the boundary condition $u(0)=\sum_{i=1}^{m-2} b_{i} u\left(\xi_{i}\right)$ implies that

$$
\min _{t \in[0, T]_{\mathbb{T}}} u(t) \geq \frac{\sum_{i=1}^{m-2} b_{i} \xi_{i}}{T-\sum_{i=1}^{m-2} b_{i}\left(T-\xi_{i}\right)} u(T) .
$$

This completes the proof.

Let $E=\mathcal{C}_{\text {ld }}[0, T]_{\mathbb{T}}$ be equipped with the norm $\|u\|=\max _{t \in[0, T]_{\mathbb{T}}}|u(t)|$. Clearly, it follows that $(E,\|u\|)$ is a Banach space. For the convenience, let

$$
\psi(s)=\phi^{-1}\left(-\frac{\sum_{i=1}^{m-2} c_{i} \int_{0}^{\xi_{i}} a(r) \nabla r}{1-\sum_{i=1}^{m-2} c_{i}}-\int_{0}^{s} a(r) \nabla r\right) .
$$

We define two cones by

$$
\begin{gathered}
P=\left\{u: u \in E, u(t) \geq 0, t \in[0, T]_{\mathbb{T}}\right\}, \\
K=\left\{u: u \in E, u(t) \text { is concave, nondecreasing, nonnegative on }[0, T]_{\mathbb{T}},\right. \\
\left.\min _{t \in[0, T]_{\mathbb{T}}} u(t) \geq \sigma\|u\|\right\},
\end{gathered}
$$

where $\sigma$ is as in Lemma 2.3. Define the operators $F: P \rightarrow E$ and $S: K \rightarrow E$ by setting

$$
\begin{aligned}
(F u)(t)= & \int_{0}^{t}(s-T) \phi^{-1}\left(-\int_{0}^{s} a(r) f(r, u(r)) \nabla r+A\right) \nabla s \\
& +\frac{\sum_{i=1}^{m-2} b_{i} \int_{0}^{\xi_{i}}(s-T) \phi^{-1}\left(-\int_{0}^{s} a(r) f(r, u(r)) \nabla r+A\right) \nabla s}{1-\sum_{i=1}^{m-2} b_{i}},
\end{aligned}
$$

where $A=-\left(\sum_{i=1}^{m-2} c_{i} \int_{0}^{\xi_{i}} a(r) f(r, u(r)) \nabla r\right) /\left(1-\sum_{i=1}^{m-2} c_{i}\right)$,

$$
(S u)(t)=\int_{0}^{t}(s-T) \varphi(s) \nabla s+\frac{\sum_{i=1}^{m-2} b_{i} \int_{0}^{\xi_{i}}(s-T) \varphi(s) \nabla s}{1-\sum_{i=1}^{m-2} b_{i}}
$$

where $\varphi(s)=\phi^{-1}\left(-\int_{0}^{s} a(r) f^{+}(r, u(r)) \nabla r+\tilde{A}\right), \tilde{A}=-\left(\sum_{i=1}^{m-2} c_{i} \int_{0}^{\xi_{i}} a(r) f^{+}(r, u(r)) \nabla r\right) /(1-$ $\left.\sum_{i=1}^{m-2} c_{i}\right)$, and $f^{+}(t, u(t))=\max \{f(t, u(t)), 0\}$. Obviously, $u$ is a solution of the BVP (1.1) if and only if $u$ is a fixed point of operator $F$. 
Lemma 2.4. $S: K \rightarrow K$ is completely continuous.

Proof. It is easy to see that $S K \subset K$ by $f^{+} \geq 0$ and Lemma 2.3. By Arzela-Ascoli theorem and Lebesgue dominated convergence theorem, we can easily prove that operator $S$ is completely continuous.

\section{Main Results}

In this section, we prove the existence of at least one positive solution to the BVP (1.1) by applying a four-functional fixed point theorem [8]. Also, by using the generalization of Leggett-Williams fixed point theorem [9], we prove the existence of at least three positive solutions for the BVP (1.1).

Let $\alpha$ and $\Psi$ be nonnegative continuous concave functionals on $P$, and let $\beta$ and $\theta$ be nonnegative continuous convex functionals on $P$, then for positive numbers $r, j, v$, and $R$, we define the following sets:

$$
\begin{gathered}
Q(\alpha, \beta, r, R)=\{x \in P: r \leq \alpha(x), \beta(x) \leq R\}, \\
U(\Psi, j)=\{x \in Q(\alpha, \beta, r, R): j \leq \Psi(x)\}, \\
V(\theta, v)=\{x \in Q(\alpha, \beta, r, R): \theta(x) \leq v\} .
\end{gathered}
$$

Theorem 3.1 (see [8]). If $P$ is a cone in a real Banach space $E, \alpha$ and $\Psi$ are nonnegative continuous concave functionals on $P, \beta$, and $\theta$ are nonnegative continuous convex functionals on $P$ and there exist positive numbers $r, j, v$, and $R$, such that

$$
A: Q(\alpha, \beta, r, R) \longrightarrow P
$$

is a completely continuous operator, and $Q(\alpha, \beta, r, R)$ is a bounded set. If

(i) $\{x \in U(\Psi, j): \beta(x)<R\} \cap\{x \in V(\theta, v): r<\alpha(x)\} \neq \emptyset$,

(ii) $\alpha(A x) \geq r$, for all $x \in Q(\alpha, \beta, r, R)$, with $\alpha(x)=r$ and $v<\theta(A x)$,

(iii) $\alpha(A x) \geq r$, for all $x \in V(\theta, v)$, with $\alpha(x)=r$,

(iv) $\beta(A x) \leq R$, for all $x \in Q(\alpha, \beta, r, R)$, with $\beta(x)=R$ and $\Psi(A x)<j$,

(v) $\beta(A x) \leq R$, for all $x \in U(\Psi, j)$, with $\beta(x)=R$,

then $A$ has a fixed point $x$ in $Q(\alpha, \beta, r, R)$.

Suppose $\tau, \eta \in \mathbb{T}$ with $0<\tau<\eta<T$. For convenience, we take the notations

$$
\begin{gathered}
\bar{w}=\frac{\sum_{i=1}^{m-2} b_{i}}{1-\sum_{i=1}^{m-2} b_{i}}, \quad N_{\eta}=\int_{0}^{\eta}(s-T) \psi(s) \nabla s, \quad N_{m-2}=\int_{0}^{\xi \xi m-2}(s-T) \psi(s) \nabla s, \\
N_{\tau}=\int_{0}^{\tau}(s-T) \psi(s) \nabla s, \quad N_{T}=\int_{0}^{T}(s-T) \psi(s) \nabla s, \\
N_{0}=\int_{0}^{\tau}(s-T) \phi^{-1}\left(-\int_{0}^{s} a(r) \nabla r\right) \nabla s .
\end{gathered}
$$


Theorem 3.2. Assume that (H1), (H2) hold if there exist constants $r, j, v, R$ with $R>\max \left\{\left(N_{T}+\right.\right.$ $\left.\left.N_{m-2} \bar{w}\right) / N_{\tau}, T / \tau\right\} j, v \geq \max \left\{\left(\left(N_{\eta}+N_{m-2} \bar{w}\right) / N_{\tau}\right) j,(\eta / \tau) r\right\}, r<j$, and suppose that $f(t, u)$ satisfies the following conditions:

(B1) $f(t, u) \leq \phi\left(R /\left(N_{T}+N_{m-2} \bar{w}\right)\right)$ for all $(t, u) \in[0, T]_{\mathbb{T}} \times[0, R]$;

(B2) $f(t, u) \geq \phi\left(r / N_{0}\right)$ for all $(t, u) \in[0, \tau]_{\mathbb{T}} \times[r, v]$;

(B3) $f(t, u) \geq 0$ for all $(t, u) \in[0, T]_{\mathbb{T}} \times[\sigma r, R]$.

Then the BVP (1.1) has a fixed point $u \in P$ such that

$$
\min _{t \in[\tau, \eta]_{\mathbb{T}}} u(t) \geq r, \quad \max _{t \in[0, T]_{\mathbb{T}}} u(t) \leq R
$$

Define maps

$$
\alpha(u)=\Psi(u)=\min _{t \in[\tau, \eta]_{\mathbb{T}}} u(t), \quad \theta(u)=\max _{t \in[\tau, \eta]_{\mathbb{T}}} u(t), \quad \beta(u)=\max _{t \in[0, T]_{\mathbb{T}}} u(t)
$$

Let $Q(\alpha, \beta, r, R), U(\Psi, j)$, and $V(\theta, v)$ be defined by (3.1).

Proof. We first show that $Q(\alpha, \beta, r, R)$ is bounded and $S: Q(\alpha, \beta, r, R) \rightarrow K$ is completely continuous. For all $u \in Q(\alpha, \beta, r, R)$, we have $\|u\|=\max _{t \in[0, T]_{\mathbb{T}}} u(t)=\beta(u) \leq R$, which means that $Q(\alpha, \beta, r, R)$ is a bounded set. According to Lemma 2.4, it is clear that $S: Q(\alpha, \beta, r, R) \rightarrow$ $K$ is completely continuous.

Let

$$
u_{0}=\frac{j}{N_{\tau}}\left(\int_{0}^{t}(s-T) \psi(s) \nabla s+\frac{\sum_{i=1}^{m-2} b_{i} \int_{0}^{\xi_{i}}(s-T) \psi(s) \nabla s}{1-\sum_{i=1}^{m-2} b_{i}}\right) .
$$

Clearly, $u_{0} \in K$. By direct calculation,

$$
\begin{aligned}
\Psi\left(u_{0}\right)= & u_{0}(\tau)=\frac{j}{N_{\tau}}\left(\int_{0}^{\tau}(s-T) \psi(s) \nabla s+\frac{\sum_{i=1}^{m-2} b_{i} \int_{0}^{\xi_{i}}(s-T) \psi(s) \nabla s}{1-\sum_{i=1}^{m-2} b_{i}}\right) \\
& \geq \frac{j}{N_{\tau}} \int_{0}^{\tau}(s-T) \psi(s) \nabla s=j, \\
\beta\left(u_{0}\right)= & \frac{j}{N_{\tau}}\left(\int_{0}^{T}(s-T) \psi(s) \nabla s+\frac{\sum_{i=1}^{m-2} b_{i} \int_{0}^{\xi_{i}}(s-T) \psi(s) \nabla s}{1-\sum_{i=1}^{m-2} b_{i}}\right) \\
\leq & \frac{j}{N_{\tau}}\left(\int_{0}^{T}(s-T) \psi(s) \nabla s+\frac{\sum_{i=1}^{m-2} b_{i} \int_{0}^{\xi_{m-2}}(s-T) \psi(s) \nabla s}{1-\sum_{i=1}^{m-2} b_{i}}\right) \\
= & \frac{j}{N_{\tau}}\left(N_{T}+\bar{\omega} N_{m-2}\right)<R,
\end{aligned}
$$




$$
\begin{aligned}
\theta\left(u_{0}\right) & =\frac{j}{N_{\tau}}\left(\int_{0}^{\eta}(s-T) \psi(s) \nabla s+\frac{\sum_{i=1}^{m-2} b_{i} \int_{0}^{\xi_{i}}(s-T) \psi(s) \nabla s}{1-\sum_{i=1}^{m-2} b_{i}}\right) \\
& \leq \frac{j}{N_{\tau}}\left(\int_{0}^{\eta}(s-T) \psi(s) \nabla s+\frac{\sum_{i=1}^{m-2} b_{i} \int_{0}^{\xi_{m-2}}(s-T) \psi(s) \nabla s}{1-\sum_{i=1}^{m-2} b_{i}}\right) \\
& =\frac{j}{N_{\tau}}\left(N_{\eta}+\bar{\omega} N_{m-2}\right) \leq v, \\
\alpha\left(u_{0}\right) & =\Psi\left(u_{0}\right) \geq j>r .
\end{aligned}
$$

So, $u_{0} \in\{u \in U(\Psi, j): \beta(u)<R\} \cap\{u \in V(\theta, v): r<\alpha(u)\}$, which means that (i) in Theorem 3.1 is satisfied.

For all $u \in Q(\alpha, \beta, r, R)$, with $\alpha(u)=r$ and $v<\theta(S u)$, we have

$$
\alpha(S u)=(S u)(\tau) \geq \frac{\tau}{\eta}(S u)(\eta)=\frac{\tau}{\eta} \theta(S u)>\frac{\tau}{\eta} v \geq r,
$$

and for all $u \in Q(\alpha, \beta, r, R)$, with $\beta(u)=R$ and $\Psi(S u)<j$, we have

$$
\beta(S u)=(S u)(T) \leq \frac{T}{\tau}(S u)(\tau)=\frac{T}{\tau} \Psi(S u)<\frac{T}{\tau} j<R
$$

Hence (ii) and (iv) in Theorem 3.1 are fulfilled.

Lastly, we consider Theorem 3.1 (iii) and (v). For all $V(\theta, v)$, with $\alpha(u)=r$,

$$
\begin{aligned}
\alpha(S u) & =\int_{0}^{\tau}(s-T) \varphi(s) \nabla s+\frac{\sum_{i=1}^{m-2} b_{i} \int_{0}^{\xi_{i}}(s-T) \varphi(s) \nabla s}{1-\sum_{i=1}^{m-2} b_{i}} \\
& \geq \int_{0}^{\tau}(s-T) \phi^{-1}\left(-\int_{0}^{s} a(r) f^{+}(r, u(r)) \nabla r-\frac{\sum_{i=1}^{m-2} c_{i} \int_{0}^{\xi_{i}} a(r) f^{+}(r, u(r)) \nabla r}{1-\sum_{i=1}^{m-2} c_{i}}\right) \nabla s \\
& \geq \int_{0}^{\tau}(s-T) \phi^{-1}\left(-\int_{0}^{s} a(r) f^{+}(r, u(r)) \nabla r\right) \nabla s \geq \frac{r}{N_{0}} \int_{0}^{\tau}(s-T) \phi^{-1}\left(-\int_{0}^{s} a(r) \nabla r\right) \nabla s=r,
\end{aligned}
$$


and for all $u \in U(\Psi, j)$, with $\beta(u)=R$,

$$
\begin{aligned}
\beta(S u) & =\int_{0}^{T}(s-T) \varphi(s) \nabla s+\frac{\sum_{i=1}^{m-2} b_{i} \int_{0}^{\xi_{i}}(s-T) \varphi(s) \nabla s}{1-\sum_{i=1}^{m-2} b_{i}} \\
& \leq \int_{0}^{T}(s-T) \varphi(s) \nabla s+\frac{\sum_{i=1}^{m-2} b_{i} \int_{0}^{\xi_{m-2}}(s-T) \varphi(s) \nabla s}{1-\sum_{i=1}^{m-2} b_{i}} \\
& \leq \frac{R}{N_{T}+N_{m-2} \bar{w}}\left(\int_{0}^{T}(s-T) \psi(s) \nabla s+\bar{w} \int_{0}^{\xi_{m-2}}(s-T) \psi(s) \nabla s\right)=R .
\end{aligned}
$$

Thus, all conditions of Theorem 3.1 are satisfied. $S$ has a fixed point $u$ in $Q(\alpha, \beta, r, R)$. Clearly, $\sigma r \leq u(t) \leq R, t \in[0, T]_{\mathbb{T}}$. By condition (B3), we have $f(t, u(t)) \geq 0, t \in[0, T]_{\mathbb{T}}$, that is, $f^{+}(t, u(t))=f(t, u(t))$. Hence, $F u=S u$. This means that $u$ is a fixed point of operator $F$. Therefore, the BVP (1.1) has at least one positive solution.

Suppose $\alpha$ and $\beta$ are two nonnegative continuous convex functionals satisfying

$$
\|x\| \leq L \max \{\alpha(x), \beta(x)\}, \quad x \in P
$$

where $L$ is a positive constant, and

$$
\Omega=\{x \in P: \alpha(x)<r, \beta(x)<l\} \neq \emptyset, \quad r>0, l>0 .
$$

Let $r>a>0, l>0$ be given, $\alpha, \beta$ nonnegative continuous convex functionals on $P$ satisfying the relation (3.12) and (3.13), and $\gamma$ a nonnegative continuous concave functional on $P$. We define the following convex sets:

$$
\begin{gathered}
P(\alpha, r ; \beta, l)=\{u \in P: \alpha(u)<r, \beta(u)<l\}, \\
\bar{P}(\alpha, r ; \beta, l)=\{u \in P: \alpha(u) \leq r, \beta(u) \leq l\}, \\
P(\alpha, r ; \beta, l ; \gamma, a)=\{u \in P: \alpha(u)<r, \beta(u)<l, \gamma(u)>a\}, \\
\bar{P}(\alpha, r ; \beta, l ; \gamma, a)=\{u \in P: \alpha(u) \leq r, \beta(u) \leq l, \gamma(u) \geq a\} .
\end{gathered}
$$

Theorem 3.3 (see [9]). Let $\mathbb{B}$ be a Banach space, $P \subset \mathbb{B}$ a cone, and $r_{2} \geq d>b>r_{1}>0, l_{2} \geq l_{1}>0$. Assume that $\alpha$ and $\beta$ are nonnegative continuous convex functionals satisfying (3.12) and (3.13), $\gamma$ is a nonnegative continuous concave functional on $P$ such that $\gamma(u) \leq \alpha(u)$ for all $u \in \bar{P}\left(\alpha, r_{2} ; \beta, l_{2}\right)$, and $A: \bar{P}\left(\alpha, r_{2} ; \beta, l_{2}\right) \rightarrow \bar{P}\left(\alpha, r_{2} ; \beta, l_{2}\right)$ is a completely continuous operator. Suppose

(i) $\left\{u \in \bar{P}\left(\alpha, d ; \beta, l_{2} ; \gamma, b\right): \gamma(u)>b\right\} \neq \emptyset, \gamma(A u)>b$ for $u \in \bar{P}\left(\alpha, d ; \beta, l_{2} ; \gamma, b\right)$;

(ii) $\alpha(A u)<r_{1}, \beta(A u)<l_{1}$ for $u \in \bar{P}\left(\alpha, r_{1} ; \beta, l_{1}\right)$;

(iii) $\gamma(A u)>b$ for $u \in \bar{P}\left(\alpha, r_{2} ; \beta, l_{2} ; \gamma, b\right)$ with $\alpha(A u)>d$. 
Then $A$ has at least three fixed points $u_{1}, u_{2}, u_{3} \in \bar{P}\left(\alpha, r_{2} ; \beta, l_{2}\right)$ with

$$
\begin{gathered}
u_{1} \in P\left(\alpha, r_{1} ; \beta, l_{1}\right), \quad u_{2} \in\left\{\bar{P}\left(\alpha, r_{2} ; \beta, l_{2} ; \gamma, b\right): \gamma(u)>b\right\}, \\
u_{3} \in \bar{P}\left(\alpha, r_{2} ; \beta, l_{2}\right) \backslash\left(\bar{P}\left(\alpha, r_{2} ; \beta, l_{2} ; \gamma, b\right) \cup \bar{P}\left(\alpha, r_{1} ; \beta, l_{1}\right)\right) .
\end{gathered}
$$

It is easy to see that for $u \in K, u(t) \geq(t / T)\|u\|, t \in[0, T]_{\mathbb{T}}$. So we get

$$
u(t) \geq \frac{\zeta}{T}\|u\|>\frac{1}{2}\|u\|, \quad t \in[\zeta, T]_{\mathbb{T}}
$$

where $\zeta$ is as in (H3). Let $\alpha, \beta$, and $\gamma$ be defined on the cone $K$ by

$$
\alpha(u)=\max _{t \in[0, T]_{\mathbb{T}}} u(t), \quad \beta(u)=\min _{t \in[0, T]_{\mathbb{T}}} u(t), \quad \gamma(u)=\min _{t \in[\zeta, T]_{\mathbb{T}}} u(t),
$$

respectively. Then it is easy to see that $\|u\|=\max \{\alpha(u), \beta(u)\}$ and (3.12), (3.13) hold. Now, for convenience, let (3.3) and $M=\int_{0}^{\zeta}(s-T) \psi(\zeta) \nabla s$.

Theorem 3.4. Assume (H1)-(H3) hold, $f(t, 0)$ is not equivalent to 0 for $t \in[0, T]_{\mathbb{T}}$. If there are positive numbers $r_{2} \geq 2 b>b>r_{1}>0, l_{2} \geq l_{1}>0$ with $b / M \leq \min \left\{r_{2} /\left(N_{T}+\right.\right.$ $\left.\left.N_{m-2} \bar{w}\right), l_{2} /\left(N_{m-2} \bar{w}\right)\right\}$, such that the following conditions are satisfied.

(C1) $0 \leq f(t, u) \leq \min \left\{\phi\left(r_{2} /\left(N_{T}+N_{m-2} \bar{w}\right)\right), \phi\left(l_{2} /\left(N_{m-2} \bar{w}\right)\right)\right\}$ for $(t, u) \in[0, T]_{\mathbb{T}} \times\left[0, r_{2}\right]$,

(C2) $f(t, u)>\phi(b / M)$ for $(t, u) \in[\zeta, T]_{\mathbb{T}} \times[b, 2 b]$,

(C3) $f(t, u)<\min \left\{\phi\left(r_{1} /\left(N_{T}+N_{m-2} \bar{w}\right)\right), \phi\left(l_{1} /\left(N_{m-2} \bar{w}\right)\right)\right\}$ for $(t, u) \in[0, T]_{\mathbb{T}} \times\left[0, r_{1}\right]$.

Then the BVP (1.1) has at least three positive solutions $u_{1}, u_{2}, u_{3}$ satisfying

$$
\begin{gathered}
\max _{t \in[0, T]_{\mathbb{T}}} u_{1}(t)<r_{1}, \quad \min _{t \in[0, T]_{\mathbb{T}}} u_{1}(t)<l_{1}, \\
b<\min _{t \in[\zeta, T]_{\mathbb{T}}} u_{2}(t) \leq \max _{t \in[0, T]_{\mathbb{T}}} u_{2}(t) \leq r_{2}, \quad \min _{t \in[0, T]_{\mathbb{T}}} u_{2}(t) \leq l_{2}, \\
\max _{t \in[0, T]_{\mathbb{T}}} u_{3}(t)<2 b, \quad \text { with } \min _{t \in[\zeta, T]_{\mathbb{T}}} u_{3}(t)<b, \min _{t \in[0, T]_{\mathbb{T}}} u_{3}(t) \leq l_{2} .
\end{gathered}
$$

Proof. By the definition of $S$, it suffices to show that the conditions of Theorem 3.3 hold with respect to the operator $S$. Firstly, we show that if the condition (C1) is satisfied, then

$$
S: \bar{K}\left(\alpha, r_{2} ; \beta, l_{2}\right) \longrightarrow \bar{K}\left(\alpha, r_{2} ; \beta, l_{2}\right)
$$


If $u \in \bar{K}\left(\alpha, r_{2} ; \beta, l_{2}\right)$, then assumption (C1) implies

$$
\begin{gathered}
f(t, u) \leq \min \left\{\phi\left(\frac{r_{2}}{N_{T}+N_{m-2} \bar{w}}\right), \phi\left(\frac{l_{2}}{N_{m-2} \bar{w}}\right)\right\}, \quad t \in[0, T]_{\mathbb{T}}, \\
\alpha(S u)=\int_{0}^{T}(s-T) \varphi(s) \nabla s+\frac{\sum_{i=1}^{m-2} b_{i} \int_{0}^{\xi_{i}}(s-T) \varphi(s) \nabla s}{1-\sum_{i=1}^{m-2} b_{i}} \\
\leq \int_{0}^{T}(s-T) \varphi(s) \nabla s+\frac{\sum_{i=1}^{m-2} b_{i} \int_{0}^{\xi_{m-2}}(s-T) \varphi(s) \nabla s}{1-\sum_{i=1}^{m-2} b_{i}} \\
\leq \frac{r_{2}}{N_{T}+N_{m-2} \bar{w}} \int_{0}^{T}(s-T) \psi(s) \nabla s+\bar{w} \int_{0}^{\xi_{m-2}}(s-T) \psi(s) \nabla s=r_{2}, \\
\beta(S u)=\frac{\sum_{i=1}^{m-2} b_{i} \int_{0}^{\xi_{i}}(s-T) \varphi(s) \nabla s}{1-\sum_{i=1}^{m-2} b_{i}} \\
\leq \frac{l_{2}}{N_{m-2} \bar{w}} \bar{w} \int_{0}^{\xi_{m-2}}(s-T) \psi(s) \nabla s=l_{2} .
\end{gathered}
$$

Therefore, (3.19) holds. In the same way, if $u \in \bar{K}\left(\alpha, r_{1} ; \beta, l_{1}\right)$, then condition (C3) implies

$$
f(t, u)<\min \left\{\phi\left(\frac{r_{1}}{N_{T}+N_{m-2} \bar{w}}\right), \phi\left(\frac{l_{1}}{N_{m-2} \bar{w}}\right)\right\}, \quad t \in[0, T]_{\mathbb{T}}
$$

As the argument above, we have $S: \bar{K}\left(\alpha, r_{1} ; \beta, l_{1}\right) \rightarrow K\left(\alpha, r_{1} ; \beta, l_{1}\right)$. Thus, condition (ii) of Theorem 3.3 holds. To check the condition (i) of Theorem 3.3, we choose

$$
\overline{u_{0}}(t)=\frac{2 b t}{T}, \quad t \in[0, T]_{\mathbb{T}}
$$

It is easy to see that $\overline{u_{0}}(t) \in \bar{K}\left(\alpha, 2 b ; \beta, l_{2} ; \gamma, b\right), \gamma\left(\overline{u_{0}}\right)>b$. Therefore, for $u \in \bar{K}\left(\alpha, 2 b ; \beta, l_{2} ; \gamma, b\right)$, there are $b \leq u(t) \leq 2 b, t \in[\zeta, T]_{\mathbb{T}}$. Hence, condition (C2) implies $f(t, u)>\phi(b / M), t \in$ $[\zeta, T]_{\mathbb{T}}$. So, by the definition of $\gamma$,

$$
\begin{aligned}
r(S u) & =\int_{0}^{\zeta}(s-T) \varphi(s) \nabla s+\frac{\sum_{i=1}^{m-2} b_{i} \int_{0}^{\xi_{i}}(s-T) \varphi(s) \nabla s}{1-\sum_{i=1}^{m-2} b_{i}} \geq \int_{0}^{\zeta}(s-T) \varphi(s) \nabla s \\
& =\int_{0}^{\zeta}(s-T) \phi^{-1}\left(-\int_{0}^{s} a(r) f^{+}(r, u(r)) \nabla r-\frac{\sum_{i=1}^{m-2} c_{i} \int_{0}^{\zeta_{i}} a(r) f^{+}(r, u(r)) \nabla r}{1-\sum_{i=1}^{m-2} c_{i}}\right) \nabla s
\end{aligned}
$$




$$
\begin{aligned}
& \geq \int_{0}^{\zeta}(s-T) \phi^{-1}\left(-\int_{0}^{\zeta} a(r) f^{+}(r, u(r)) \nabla r-\frac{\sum_{i=1}^{m-2} c_{i} \int_{0}^{\xi_{i}} a(r) f^{+}(r, u(r)) \nabla r}{1-\sum_{i=1}^{m-2} c_{i}}\right) \nabla s \\
& >\frac{b}{M} \int_{0}^{\zeta}(s-T) \phi^{-1}\left(-\int_{0}^{\zeta} a(r) \nabla r-\frac{\sum_{i=1}^{m-2} c_{i} \int_{0}^{\xi_{i}} a(r) \nabla r}{1-\sum_{i=1}^{m-2} c_{i}}\right) \nabla s=b .
\end{aligned}
$$

Hence, condition (i) of Theorem 3.3 holds. We finally prove that (iii) in Theorem 3.3 holds. In fact, for $u \in \bar{K}\left(\alpha, 2 b ; \beta, l_{2} ; \gamma, b\right)$ with $\alpha(S u)>2 b$, we have $\gamma(S u)>b$. Thus from Theorem 3.3, the BVP (1.1) has at least three fixed points $u_{1}, u_{2}, u_{3} \in \bar{K}\left(\alpha, r_{2} ; \beta, l_{2}\right)$. Clearly, $0 \leq u_{1}(t) \leq r_{1}$, $\sigma b<u_{2}(t) \leq r_{2}, 0 \leq u_{3}(t) \leq 2 b, t \in[0, T]_{\mathbb{T}}$. By condition $(C 1)$, we have $f(t, u(t)) \geq 0, t \in[0, T]_{\mathbb{T}}$, that is, $f^{+}(t, u(t))=f(t, u(t))$. Hence, $F u=S u$. This means that $u_{1}, u_{2}, u_{3}$ are fixed points of operator $F$. Therefore, the BVP (1.1) has at least three positive solutions.

\section{Example}

Example 4.1. Let $\mathbb{T}=[0,1 / 2] \cup\{0.6\} \cup[0.7,1]$. If we choose $T=1, a(t)=1, b_{1}=1 / 3, c_{1}=1 / 2$, $\xi_{1}=1 / 4$ in the boundary value problem (1.1), then we have the following BVP on time scale $\mathbb{T}$ :

$$
\begin{gathered}
\left(\phi\left(u^{\Delta \nabla}(t)\right)\right)^{\nabla}+f(t, u(t))=0, \quad t \in[0,1]_{\mathbb{T}}, \\
u(0)=\frac{1}{3} u\left(\frac{1}{4}\right), \quad u^{\Delta}(1)=0, \quad \phi\left(u^{\Delta \nabla}(0)\right)=\frac{1}{2} \phi\left(u^{\Delta \nabla}\left(\frac{1}{4}\right)\right),
\end{gathered}
$$

where

$$
\begin{gathered}
\phi(u)= \begin{cases}u, & u \leq 0, \\
u^{2}, & u>0,\end{cases} \\
f(t, u)= \begin{cases}95(u-0.0001)+0.0007, & {[0,1]_{\mathbb{T}} \times[0,0.0001],} \\
0.0007, & {[0,1]_{\mathbb{T}} \times[0.0001, \infty) .}\end{cases}
\end{gathered}
$$

Set $\tau=1 / 3, \eta=0.6$, by calculation,

$$
\begin{gathered}
\bar{w}=\frac{1}{2}, \quad N_{T}=\frac{1721}{6000}, \quad N_{m-2}=\frac{31}{384}, \quad N_{\tau}=\frac{73}{648}, \quad N_{0}=\frac{7}{162}, \\
N_{\eta}=\frac{2533}{12000}, \quad \sigma=\frac{1}{9},
\end{gathered}
$$


and let $r=0.001, j=r+10^{-4}, R=0.012$, and $v=0.0035$ with $0.012>0.0033,0.0035 \geq$ 0.002455 . Clearly, we can verify that the conditions in Theorem 3.2 are fulfilled. Thus, by Theorem 3.2, the BVP (4.1) has a fixed point $u$ such that

$$
\min _{t \in[1 / 3,0.6]_{\mathbb{T}}} u(t) \geq 0.001, \quad \max _{t \in[0,1]_{\mathbb{T}}} u(t) \leq 0.012
$$

Example 4.2. Let $\mathbb{T}=[0,1] \cup\{1.2\} \cup\{1.5\} \cup[1.8,2]$. If we choose $T=2, a(t)=1, b_{1}=1 / 4$, $c_{1}=1 / 3, \xi_{1}=2 / 3$ in the boundary value problem (1.1), then we have the following BVP on time scale $\mathbb{T}$ :

$$
\begin{gathered}
\left(\phi\left(u^{\Delta \nabla}(t)\right)\right)^{\nabla}+f(t, u(t))=0, \quad t \in[0,2]_{\mathbb{T}} \\
u(0)=\frac{1}{4} u\left(\frac{2}{3}\right), \quad u^{\Delta}(2)=0, \quad \phi\left(u^{\Delta \nabla}(0)\right)=\frac{1}{3} \phi\left(u^{\Delta \nabla}\left(\frac{2}{3}\right)\right),
\end{gathered}
$$

where

$$
\begin{gathered}
\phi(u)= \begin{cases}u, & u \leq 0, \\
u^{2}, & u>0,\end{cases} \\
f(t, u)= \begin{cases}\frac{1}{80} t+\frac{1}{10} u^{3}, & t \in[0,2]_{\mathbb{T}}, u \in[0,4], \\
\frac{1}{80} t+\frac{8}{5} u, & t \in[0,2]_{\mathbb{T}}, u \in(4,200], \\
\frac{1}{80} t+(520-u), & t \in[0,2]_{\mathbb{T}}, u \in(200, \infty) .\end{cases}
\end{gathered}
$$

Obviously the hypotheses $(\mathrm{H} 1),(\mathrm{H} 2)$ hold and $f(t, 0)$ is not equivalent to 0 on $[0,2]_{\mathbb{T}}$. By simple calculations, we have

$$
\zeta=1.2, \quad \bar{w}=\frac{1}{3}, \quad N_{T}=1.859, \quad N_{m-2}=0.716, \quad M=2.5398 .
$$

If we choose $r_{2}=200, b=2, r_{1}=1 / 2$, and $l_{2}=80, l_{1}=20$, then $f(t, u)$ satisfies

(1) $0 \leq f(t, u) \leq 320.025 \leq \min \left\{\phi\left(r_{2} /\left(N_{T}+N_{m-2} \bar{w}\right)\right), \phi\left(l_{2} / N_{m-2} \bar{w}\right)\right\}=9090$ for $(t, u) \in$ $[0,2]_{\mathbb{T}} \times[0,200]$

(2) $f(t, u) \geq 0.815>\phi(b / M)=0.62$ for $(t, u) \in[1.2,2]_{\mathbb{T}} \times[2,4]$,

(3) $f(t, u) \leq 0.0375<\min \left\{\phi\left(r_{1} /\left(N_{T}+N_{m-2} \bar{w}\right)\right), \phi\left(l_{1} /\left(N_{m-2} \bar{w}\right)\right)\right\}=0.0568$. 
So, all conditions of Theorem 3.4 hold. Thus by Theorem 3.4, the BVP (4.5) has at least three positive solutions $u_{1}, u_{2}, u_{3}$ such that

$$
\begin{gathered}
\max _{t \in[0,2]_{\mathbb{T}}} u_{1}(t)<\frac{1}{2}, \quad \min _{t \in[0,2]_{\mathbb{T}}} u_{1}(t)<20, \\
2<\min _{t \in[1.2,2]_{\mathbb{T}}} u_{2}(t) \leq \max _{t \in[0,2]_{\mathbb{T}}} u_{2}(t) \leq 200, \quad \min _{t \in[0,2]_{\mathbb{T}}} u_{2}(t) \leq 80, \\
\max _{t \in[0,2]_{\mathbb{T}}} u_{3}(t)<4, \quad \text { with } \min _{t \in[1.2,2]_{\mathbb{T}}} u_{3}(t)<2, \min _{t \in[0,2]_{\mathbb{T}}} u_{3}(t) \leq 80 .
\end{gathered}
$$

\section{References}

[1] S. Hilger, "Analysis on measure chains-a unified approach to continuous and discrete calculus," Results in Mathematics, vol. 18, no. 1-2, pp. 18-56, 1990.

[2] M. Bohner and A. Peterson, Dynamic Equations on Time Scales, An Introduction with Applications, Birkhäuser, 2001.

[3] M. Bohner and A. Peterson, Advances in Dynamic Equations on Time Scales, Birkhäuser, 2003.

[4] J. Adem and M. Moshinsky, "Self-adjointness of a certain type of vectorial boundary value problems," Boletín de la Sociedad Matemática Mexicana, vol. 7, pp. 1-17, 1950.

[5] V. A. Ilin and E. I. Moiseev, "Nonlocal boundary value problem of the second kind for a SturmCLiouville operator," Difference Equation, vol. 23, pp. 979-987, 1987.

[6] Z. Bai, W. Ge, and Y. Wang, "Multiplicity results for some second-order four-point boundary-value problems," Nonlinear Analysis. Theory, Methods \& Applications A, vol. 60, no. 3, pp. 491-500, 2005.

[7] Y. Zou, Q. Hu, and R. Zhang, "On numerical studies of multi-point boundary value problem and its fold bifurcation," Applied Mathematics and Computation, vol. 185, no. 1, pp. 527-537, 2007.

[8] R. Avery, J. Henderson, and D. O'Regan, "Four functionals fixed point theorem," Mathematical and Computer Modelling, vol. 48, no. 7-8, pp. 1081-1089, 2008.

[9] Z. Bai and W. Ge, "Existence of three positive solutions for some second-order boundary value problems," Computers \& Mathematics with Applications, vol. 48, no. 5-6, pp. 699-707, 2004.

[10] R. W. Leggett and L. R. Williams, "Multiple positive fixed points of nonlinear operators on ordered Banach spaces," Indiana University Mathematics Journal, vol. 28, no. 4, pp. 673-688, 1979.

[11] T.-T. Sun, L.-L. Wang, and Y.-H. Fan, "Existence of positive solutions to a nonlocal boundary value problem with $p$-Laplacian on time scales," Advances in Difference Equations, Article ID 809497, 15 pages, 2010.

[12] J. Liu and H.-R. Sun, "Multiple positive solutions for $m$-point boundary value problem on time scales," Boundary Value Problems, vol. 2011, Article ID 591219, 2011.

[13] F. Xu and Z. Meng, "The existence of positive solutions for third-order $p$-Laplacian $m$-point boundary value problems with sign changing nonlinearity on time scales," Advances in Difference Equations, vol. 2009, Article ID 169321, 14 pages, 2009.

[14] D. Ji, Y. Tian, and W. Ge, "Positive solutions for one-dimensional $p$-Laplacian boundary value problems with sign changing nonlinearity," Nonlinear Analysis. Theory, Methods E Applications A, vol. 71, no. 11, pp. 5406-5416, 2009.

[15] Y. Sang and H. Su, "Positive solutions of nonlinear third-order $m$-point BVP for an increasing homeomorphism and homomorphism with sign-changing nonlinearity," Journal of Computational and Applied Mathematics, vol. 225, no. 1, pp. 288-300, 2009.

[16] Y. Zhu and J. Zhu, "The multiple positive solutions for $p$-Laplacian multipoint BVP with sig changing nonlinearity on time scales," Journal of Mathematical Analysis and Applications, vol. 344, no. 2, pp. 616626, 2008.

[17] Y. Sang, H. Su, and F. Xu, "Positive solutions of nonlinear $m$-point BVP for an increasing homeomorphism and homomorphism with sign changing nonlinearity on time scales," Computers $\mathcal{E}$ Mathematics with Applications, vol. 58, no. 2, pp. 216-226, 2009. 
[18] K. Q. Lan, "Multiple positive solutions of semilinear differential equations with singularities," Journal of the London Mathematical Society, vol. 63, no. 3, pp. 690-704, 2001.

[19] D. J. Guo and V. Lakshmikantham, Nonlinear Problems in Abstract Cones, vol. 5, Academic Press, Boston, Mass, USA, 1988. 


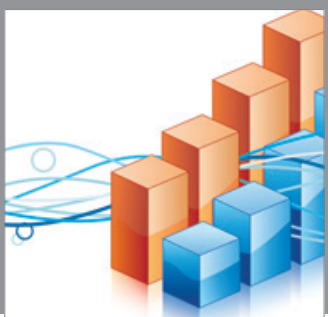

Advances in

Operations Research

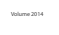

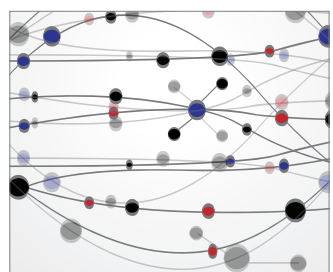

\section{The Scientific} World Journal
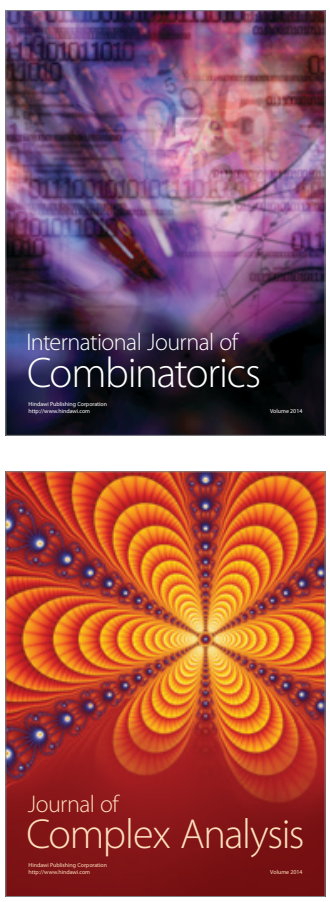

International Journal of

Mathematics and

Mathematical

Sciences
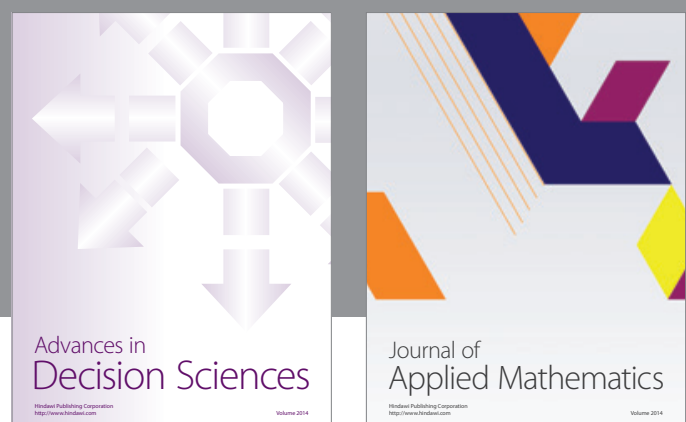

Journal of

Applied Mathematics
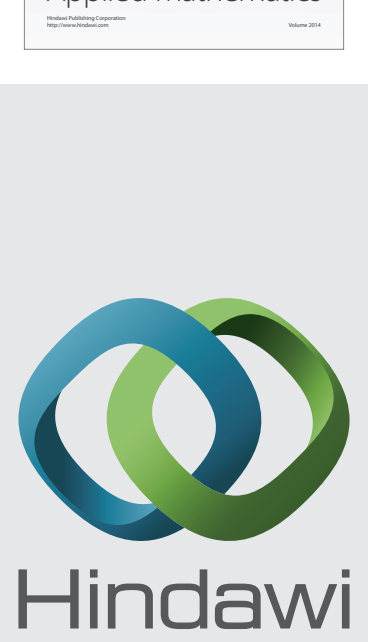

Submit your manuscripts at http://www.hindawi.com
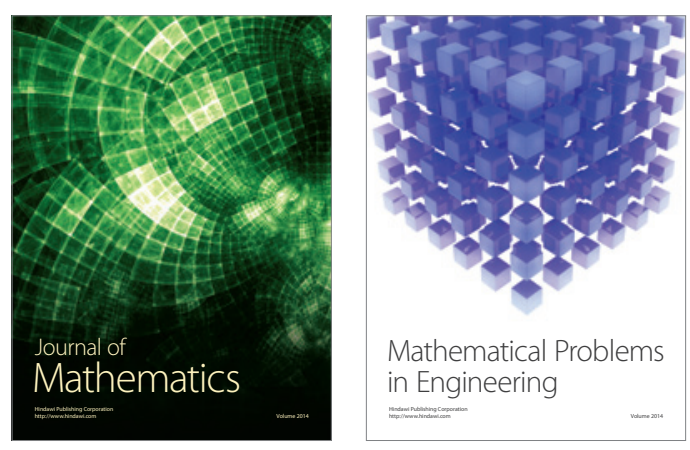

Mathematical Problems in Engineering
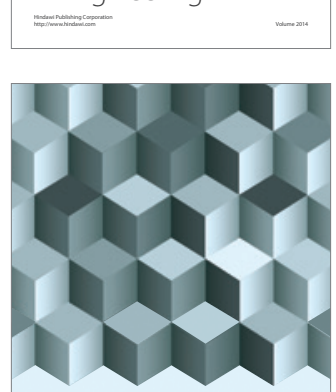

Journal of

Function Spaces
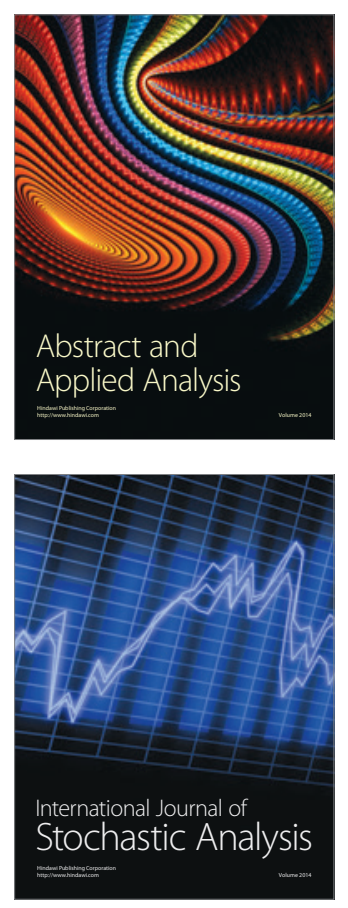

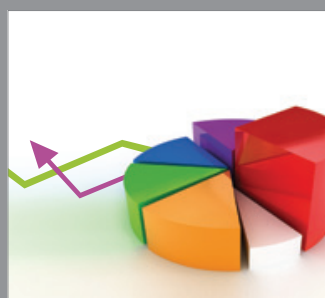

ournal of

Probability and Statistics

Promensencen
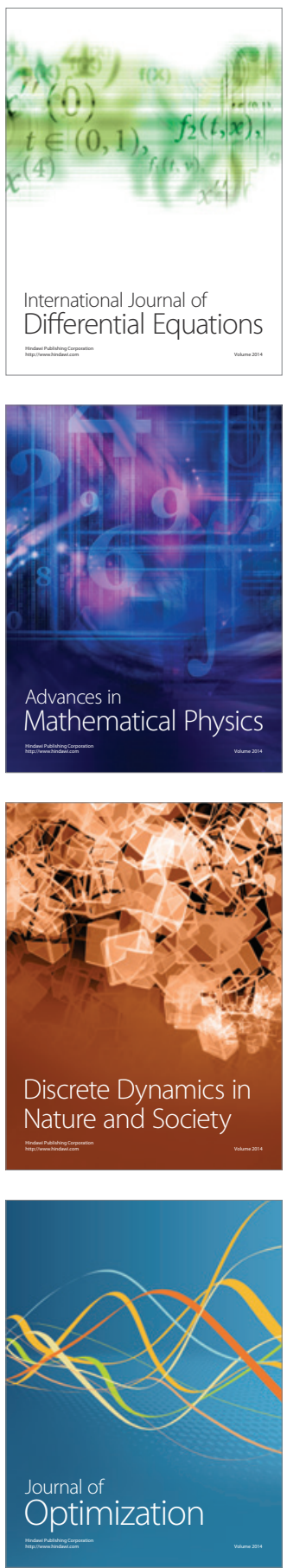